\title{
Ureteroscopic holmium:YAG laser endopyelotomy is effective in distinctive ureteropelvic junction obstructions
}

\author{
Zhong Wu*, Chenchen Feng*, Qiang Ding, Haowen Jiang, Yuanfang Zhang \\ Department of Urology, Huashan Hospital, Fudan University, Shanghai, PR China \\ *Authorships of equal contribution.
}

Videosurgery and other miniinvasive techniques 2011; 6 (3): 144-149 DOI: 10.5114/wiitm.2011.24692

\begin{abstract}
Aim: To evaluate the effectiveness and safety of holmium:YAG (Ho:YAG) laser endopyelotomy in distinctive ureteropelvic junction obstructions (UPJO) with distinctive aetiologies.

Material and methods: Thirty-one patients diagnosed with UPJO of distinctive causes were included. Aetiology consisted of 7 congenital UPJO, 10 post-pyeloplasty UPJO, 7 post-lithotomy obstructions, 4 ureteropelvic junction obstructions post-extracorporeal shockwave lithotripsy stenoses and 3 post-ureteroscopic lithotriptic UPJO. Retrograde ureteroscopic Ho:YAG laser endopyelotomy was performed in all patients. Operation related parameters were studied. Results: Average procedure duration was 46 min. Mean discharge was 1.81 days. There was no notable complication such as perforation or haemorrhage. All patients were followed for at least 12 months. The single success rate was 80.6\%, leaving 6 patients undergoing secondary endopyelotomy, among whom 4 were successful while 2 required an open approach. The overall success rate was $93.5 \%$. Failed pyeloplasty UPJO is more disposed to restenosis $(p=0.0075)$. Inversely implanted ureteral stent yielded a higher success rate $(p=0.0158)$.

Conclusions: Ho:YAG laser endopyelotomy is a safe, minimally invasive approach effective in both primary and secondary UPJO treatments. Implantation of inversed ureteral stents can be more beneficial.
\end{abstract}

Key words: ureteropelvic junction obstruction, ureteroscopic, endopyelotomy, laser.

\section{Introduction}

Rapid development of minimally invasive approaches has enabled minimized trauma, swift convalescence and more exquisite surgical manipulations with outcomes equivalent to open procedures. Current treatment of ureteropelvic junction obstructions (UPJO) is no longer constrained with open, laparoscopic or robot-assisted pyeloplasties. Endoscopic management of UPJO has been reported in a number of centres with either an antegrade (percutaneous) or a retrograde approach. The latter appears more appealing since it brings even less invasiveness by using a natural human orifice. Amid all the modalities a ureteroscope equipped with a Holmium:Yttrium-Aluminium-Garnet (Ho:YAG) laser is widely applied due to its precision and minimal thermal spread in tissue cutting. However, most present reports on Ho:YAG endopyelotomy consist of small series of patients with limited aetiologies [1-6]. More accumulated data and technical tips should be gathered before establishing an instructive guideline for the procedure.

Our centre has been practising ureteroscopic laser lithotripsy for over 2000 casus which encouraged us to carry out laser endopyelotomies in 31 patients 
with UPJO, among whom the aetiology varied from congenital malformation to extracorporeal shockwave lithotripsy (SWL) complication. Also, we herein report a manipulation trick in stent placement which results in exciting outcomes.

\section{Material and methods}

\section{General information}

From October 2004 to January 2010, a total of 31 patients diagnosed with UPJO underwent ureteroscopic endopyelotomy with Ho:YAG laser at our institute. There were 19 males (61.3\%) and 12 females $(38.7 \%)$ at the mean age of 43.5 years, ranging from 19 years to 67 years. Obstructions on left and right sides were in 18 and 13 cases respectively with an average stenosis length of $1.3 \mathrm{~cm}(0.7 \mathrm{~cm}$ to $2.0 \mathrm{~cm})$. Aetiology included congenital obstruction in 7 cases (22.6\%), recurrent stenosis after primary UPJO pyeloplasty in 10 cases (32.3\%), postoperative obstruction of ureteropelvic junction (UPJ) lithotomy in 7 cases (22.6\%), post-SWL obstruction of UPJ calculi in 4 cases $(12.9 \%)$ and postoperative stenosis of ureteroscopic lithotripsy in 3 cases (9.6\%). All patients were confirmed with the diagnosis by more than one auxiliary examination including ultrasound, intravenous pyelography (IVP) or retrograde pyelography (RP), diuretic renogram, computed tomography (CT) and Magnetic Resonance Urography (MRU), among which the pyelogram was imperative. Exclusion criteria included obstructions longer than $2 \mathrm{~cm}$, ipsilateral upper urinary calculi and suspicion of crossing vasculature at the posterior or lateral wall(s) of the obstruction, identified by any of the radiographic studies. Preoperative infection was maximally controlled with oral or intravenous antibiotics. This study conformed to the provisions of the Declaration of Helsinki, 2004.

\section{Techniques and follow-ups}

Patients were placed in the lithotomy position under general or epidural anaesthesia. Intraoperative pyelogram was not performed to minimize the operative time. A 0.035 inch guidewire was introduced for passage of the ureteroscope through the ureteral orifice. No balloon dilation was used and fluoroscopic monitoring was maintained throughout the procedure. The Wolf 8/9.8F semi-rigid ureteroscope was initially applied with caution for sharp distortions and transmitted pulsations at the UPJ. Significant kinking required a second wire to diminish the torsion. Nonetheless, intraoperative change of a Storz flexible ureteroscope was immediately exerted if the doublerailed guidance was still insufficient without forced or duplicate attempts with the semi-rigid device. Usually the passage was impeded below the UPJ (Figure 1).

Delivery of the holmium laser (Versa Pulse Select) was conducted through a $365-\mu \mathrm{m}$ or $200-\mu \mathrm{m}$ fibre at the initial energy of $1 \mathrm{~J}$ and pulse rate of $10 \mathrm{~Hz}$. A retrograde cutting pattern was achieved by slowly advancing the ureteroscope into the renal pelvis. The incision was commenced at approximately $0.5 \mathrm{~cm}$ below the obstruction and was performed over the obstruction with another $0.5 \mathrm{~cm}$ distance. Completely under direct visual monitoring, the obstruction was incised lamina by lamina. The ureteroscope could be used to assist the procedure by gentle passage into the pelvis and mild lateral parting of the cut edges. Haemorrhage encountered was cauterized by the defocused laser beam and the incision was deepened until the peripelvic and periureteral fat was perceived. The technique of marsupialisation was not applied since high insertion was not identified in any case. The procedure was completed with passage of the ureteroscope through the incised part into the pelvis for dilation. In the cases in which a flexible ureteroscope was used for incision, the eventual dilation was performed in order by the flexible and semirigid apparatus respectively. A $7 \mathrm{~F}$ endopyelotomy

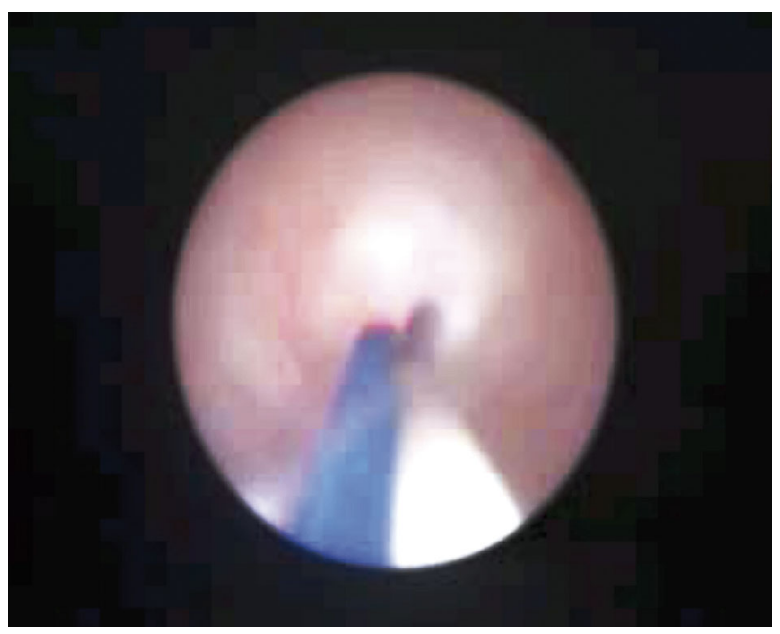

Figure 1. Ureteroscopic view of a post-pyeloplasty UPJO allowing only the passage of 0.035 inch guidewire (white). Laser fibre (blue) with aim beam stand-by 


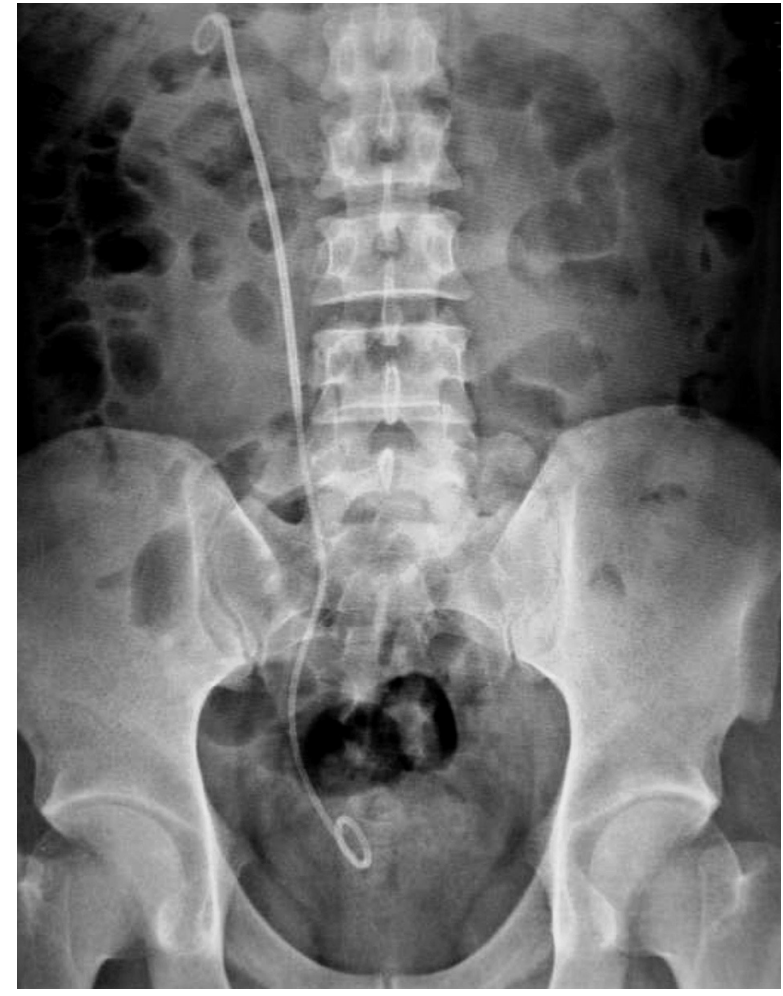

Figure 2. KUB revealing implanted double J stent in appropriate position on postoperative day 1

stent was implanted in the ureter and was left indwelling for 4 to 8 weeks with urethral catheterization of 1 to 2 days. Stenting was placed in the common position with the tapering end in the pelvis of 11 procedures whereas it was implanted inversely in 26 procedures (including secondary intervention).

Postoperative follow-ups included instant X-ray of kidney, ureter, and bladder (KUB) on day 1 and fortnightly; IVP, renal function, ultrasound and renogram examination every 3 months and for at least 1 year if no secondary intervention was needed; and cystoscopic removal of the ureter stent pending radiographic feedbacks, with which all patients complied. The procedure was considered successful when all criteria, namely alleviation of preoperative symptoms, stabilization of the renal function changes and radiographic evidence of significant degraded hydronephrosis after stent removal, were met after 12 months of the operation.

Data were processed with SPSS Statistics ver. 17. Central tendencies were expressed as means. Fisher's exact test was applied for comparison study. All results were 2 -tailed and $p<0.05$ was accepted as statistically significant.

\section{Results}

Subjects involved in the present study were all detected with grade 3 (13) and grade 4 (18) hydronephrosis preoperatively. Patients, except for those diagnosed with congenital UPJO or post-SWL stenosis, all had a history of ureteral stent placement. Five patients experienced intraoperative change to a flexible device. The mean operation time was $46 \mathrm{~min}$ (32 min to $61 \mathrm{~min}$ ). There was no significant intraoperative complication such as uncontrolled bleeding or perforation. Coagulation with the defocused laser beam was exerted times per site. All patients were incised adequately in the operation with successful stent indwelling (Figure 2).

Postoperative evaluation is partly summarized in Table I. Patients regardless of aetiology were discharged after 1.81 days on average (Table I). Instant postoperative complications were minor, without UPJ haematoma, vesicoureteral reflux or displacement of the double J stent. Due to urethral catheterization, slight urinary infection was noted in 11 patients who were cured by oral antibiotics (Table I). Pyelonephritis was not noted in any of the cases. Restenosis in 6 patients was all observed in the first 3 postoperative months of follow-up. Diagnoses were made mainly on radiographic evidence as the complaints were indifferent and renal function was not revealed to be deteriorating. The recurrent cases comprised 5 failed pyeloplasties and 1 complication of SWL (Table I). By comparison, failed pyeloplasty yielded a significantly higher tendency of single endopyelotomy failure than other causes included in the present study (Table 1). A tendency with SWL on the other hand was not notable (Table I). In the 6 failed cases a second endopyelotomy was performed in accordance with the methodologies described in Techniques and Follow-ups. The operation was successful in 4 patients, leaving 2 subjects (1 failed pyeloplasty and 1 SWL complication) with severe obstruction requiring open surgery.

Follow-ups of all patients, including reoperated ones, were conducted for 12 months minimum. The mean period was 11.8 months (3 to 18 months), resulting from termination of follow-up in 6 patients when secondary intervention was mandatory and in 2 patients when tertiary surgery was performed. The average duration of ureteral stent placement was 6.0 weeks. Subjects with inversely implanted stents achieved a significantly higher success rate than those 
with ortho-positioned ones (Table I). The single operational success rate was $80.6 \%$ and the overall success rate was $93.5 \%$ in our series (Table I, Figure 3).

\section{Discussion}

Attempts of an intraluminal approach of treating UPJ obstruction date from 1983, when J.E.A. Wickham performed a cold-knife incision by an antegrade approach. Since then, development of endoscopic therapy for UPJO has chiefly advanced in 2 aspects: improvement of the high resolution visualisation system that enables intraoperative monitoring and a variety of ablative tools for more efficient and safer tissue cutting. Thus far, endopyelotomy has become the first line treatment for UPJO in many centres, among which the most commonly practised combination is Ho:YAG laser with a retrograde passage $[4,7,8]$. Delivery of the laser consists of 2 parts. Holmium, a rare earth element, is used as the active medium and an yttrium-aluminium-garnet (YAG) crystal is for conduction. This solid-state system emits a pulsed laser beam of $2140 \mathrm{~nm}$ in wavelength with a pulse duration ranging from $0.25 \mathrm{~s}$ to $0.35 \mathrm{~s}$. Such duration enables an elongated cavitation bubble to generate a weaker shockwave than most short-pulsed lasers. Evidence of stone vaporization in Ho:YAG lithotripsy indicates a photothermal mechanism, which in turn validates its application in tissue cutting as tissues are composed mainly of water. The energy is thus mainly absorbed within the surface, leaving a superficial incision or ablation. Thermal injury with the ablation ranges from 0.5 to $1.0 \mathrm{~mm}$ [9-11]. With the presence of clear fluoroscopic monitoring, cutting with the Ho:YAG laser is very delicate and totally controllable for thin layer slicing.

Although the procedure is now practised worldwide, a normalized manipulation guideline has not yet emerged. Authors generally report their experiences conforming to certain criteria. Firstly, patients considered for an endopyelotomy should be excluded if they have long obstructions $(>2 \mathrm{~cm})$, stenosis with crossing vasculature, or with severe hydronephrosis. Such patients may benefit from laparoscopic or robot-assisted pyeloplasty due to increase of pelvic volume. Secondly, the incision is recommended to be posterolateral and through the fibrous tissue in recurrent cases. However, in our series, the dictum was not strictly followed as under completely lucid visualisation it is possible to control the cutting
Table I. Postoperative evaluations of ureteroscopic Ho:YAG laser endopyelotomy

\begin{tabular}{|lc|}
\hline Inpatient duration* & 1.81 days (mean) \\
\hline 1 day (\%) & $13(35.1 \%)$ \\
\hline 2 days (\%) & $18(48.7 \%)$ \\
\hline 3 days (\%) & $6(16.2 \%)$ \\
\hline Complications (\%) & $17(55 \%)$ \\
\hline Urinary infection (\%) & $11(35.5 \%)$ \\
\hline Re-obstruction (\%) & $6(19.4 \%)$ \\
\hline \begin{tabular}{l} 
Post pyeloplasty of primary UPJO (\%) \\
\hline Other preoperative diagnoses (\%)
\end{tabular} & $5 / 10(50.0 \%)$ \\
\hline \begin{tabular}{l} 
Value of $p$ \\
\hline Post-SWL lithotripsy (\%)
\end{tabular} & $1 / 21(4.8 \%)$ \\
\hline Other preoperative diagnoses (\%) & $5 / 28(17.9 \%)$ \\
\hline $\begin{array}{l}\text { Value of } p \\
\text { Stent position related success rate }\end{array}$ & 0.4314 \\
\hline \begin{tabular}{l} 
Ortho-position (\%) \\
\hline Inverted position (\%)
\end{tabular} \\
\hline Value of $p$ & $6 / 11^{\star}(54.5 \%)$ \\
\hline Overall success rate (\%) & $24 / 26^{*}(92.3 \%)$ \\
\hline
\end{tabular}

*Six patients with secondary operation after primary failure also included. Number without unit indicates subject number

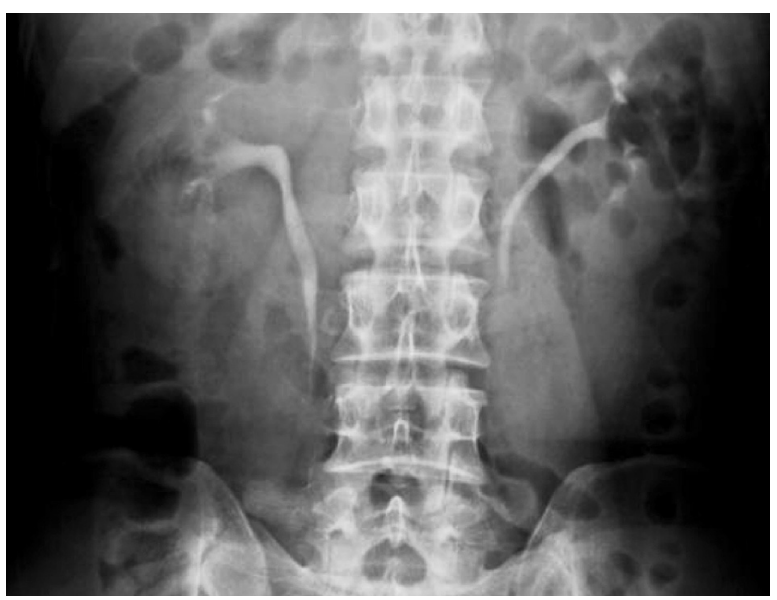

Figure 3. Follow-up IVP done 12 months after endopyelotomy demonstrating satisfactory excretion of the contrast through the right UPJ 
depth and cauterize slight bleeding until the peripelvic fat is seen. We suggest that the incision should start from where the revelation is better so the possible damage of the guide wire or profound cutting can be maximally avoided. As for whether patients with high insertion of the UPJO are contradicted for the operation remains controversial due to previous poor results. Nonetheless, with careful marsupialisation technique, some authors have reported satisfactory outcomes $[1,12]$. Similar technique is also successfully applied in a case report concerning a lower pole moiety UPJO in a partially duplicated collecting system [13].

In carefully selected patients, ureteroscopic Ho:YAG laser endopyelotomy is proven to be highly effective and beneficial. In contrast to the antegrade approach, which is more disposed to postoperative re-stenosis and renal lithiasis, the retrograde procedure is additionally convenient with less invasiveness. The availability of a flexible ureteroscope further facilitates the accessibility to the UPJ. The Ho:YAG laser is also reported to exceed the effectiveness of the balloon catheter (Acucise ${ }^{T M}$ ) [14]. Some doubts arose when Rassweiler et al. [15] conducted a comparative study within a large series of patients undergoing endopyelotomy and laparoscopic pyeloplasty. They point out that with the goal of achieving the optimal long-term success rate of open surgery $(93 \%$ to $97 \%$ ), which is considered the gold standard treatment, endopyelotomy yields a lower long-term success rate, requires more particular inclusion criteria, and is less effective for extrinsic stenosis in comparison with the laparoscopic approach, which is parallel to open dismembered pyeloplasty in outcomes. Interestingly, most reports on endopyelotomy cover the aetiology of failed pyeloplasty resulting from open or laparoscopic management $[16,17]$ and have revealed an effective remedy for such cases.

The focus of this dispute concerning endopyelotomy remains on how broadly should the patients be included and how long should the follow-ups last to be regarded as successful treatment. Hibi et al. [18] reported a success rate of $80 \%$ at 18 months when all stricture recurrence can be observed at average follow-up of 60.5 months. Doo et al. [19] reported a declined success rate of endopyelotomy from $87.8 \%$ at 6 months to $61.6 \%$ at 60 months. They recommend a follow-up as long as 36 months although most recurrent stenoses can be observed within
1 year. In our series, however, the initial manipulation is not as successful within patients of failed pyeloplasty (Table I). This could be due to both the unfamiliarity of the procedure and the substantially higher recurrence rate of the aetiology. In line with most reports, the average inpatient duration and operation time in our series is short. In reference to the principles suggested by Davis [20] that success of endopyelotomy depends on complete incision and efficient drainage, and the ureter wall regenerates on a tutor probe 6 weeks after a longitudinal incision, we designate a 6-week basis of stent removal. Predated extraction and prolonged placement of stents are due to complications (e.g. infection, lithiasis) and reoperations respectively. All patients including reoperated ones were followed at least for 12 months during which period radiographic and biochemical evidence was taken as importantly as patients' symptoms. Although the single operational success rate is average among distinctive reports, the overall success rate increases to $93.5 \%$ regardless of obstruction causes, which can be the result of a technique trick by inversely placing the ureteral stent (Table I). The inversely implanted stent may provide more adequate dilation within the same period and can thus lead to better outcomes when removal is necessary.

Back to the opposing respects of endopyelotomy, we can actually see the increasing effectiveness and improving techniques reported from centre to centre. The presence of both approaches may, in lieu of replacing one another, compensate for each other's weak points. Laser endopyelotomy is perfectly compatible with laparoscopic, open or even robot-assisted management in case of failure. Our experience of achieving high effectiveness with Ho:YAG laser endopyelotomy among distinctive aetiologies may contribute in part to the recognition and popularization of the approach.

\section{Conclusions}

Ureteroscopic Ho:YAG laser endopyelotomy brings little trauma, and a short stay without haemorrhagic complications. Obstructions due to failed pyeloplasty yield a higher recurrence rate than other causes such as congenital UPJO or lithotriptic complications. The single success rate is $80.6 \%$ whereas the overall success rate is $93.5 \%$. An inversely implanted ureteral stent is associated with a higher success rate. 


\section{Acknowledgments}

Drs. Zhong Wu, Chenchen Feng, Qiang Ding, Haowen Jiang and Yuanfang Zhang have no conflicts of interest or financial ties to disclose.

\section{References}

1. Matin SF, Yost A, Streem SB. Ureteroscopic laser endopyelotomy: a single-center experience. J Endourol 2003; 17: 401-4.

2. Biyani CS, Cornford PA, Powell CS. Ureteroscopic endopyelotomy with the Holmium:YAG laser. mid-term results. Eur Urol 2000; 38: 139-43.

3. Gerber GS, Kim JC. Ureteroscopic endopyelotomy in the treatment of patients with ureteropelvic junction obstruction. Urology 2000; 55: 198-202.

4. Giddens JL, Grasso M. Retrograde ureteroscopic endopyelotomy using the holmium:YAG laser. J Urol 2000; 164: 1509-12.

5. Conlin MJ, Bagley DH. Ureteroscopic endopyelotomy at a single setting. J Urol 1998; 159: 727-31.

6. Renner C, Frede T, Seemann O, Rassweiler J. Laser endopyelotomy: minimally invasive therapy of ureteropelvic junction steno sis. J Endourol 1998; 12: 537-44.

7. Nakada SY, Johnson M. Ureteropelvic junction obstruction. Retrograde endopyelotomy. Urol Clin North Am 2000; 27: 677-84.

8. Van Cangh PJ, Nesa S, Tombal B. The role of endourology in Ureteropelvic junction obstruction. Curr Urol Rep 2001; 2: 149-53.

9. Wollin TA, Denstedt JD. The holmium laser in urology. J Clin Laser Med Surg 1998; 6: 13-20.

10. Vassar GJ, Chan KF, Teichman JM, et al. Holmium: YAG lithotripsy: photothermal mechanism. J Endourol 1999; 13: 181-90.

11. Dushinski JW, Lingeman JE. High-speed photographic evaluation of holmium laser. J Endourol 1998; 12: 177-81.

12. Thomas R, Monga M, Klein EW. Ureteroscopic retrograde endopyelotomy for management of ureteropelvic junction obstruction. J Endourol 1996; 10: 141-5.

13. Taniguchi M, Kamei S, Takeuchi T, et al. Successful management of lower pole moiety ureteropelvic junction obstruction in a partially duplicated collecting system using retrograde endoureteropyelotomy with the Holmium:YAG laser. Int J Urol 2005; 12: 313-5.

14. el-Nahas AR, Shoma AM, Eraky I, et al. Prospective, randomized comparison of ureteroscopic endopyelotomy using holmium: YAG laser and balloon catheter. J Urol 2006; 175: 614-8.

15. Rassweiler JJ, Subotic S, Feist-Schwenk M, et al. Minimally invasive treatment of ureteropelvic junction obstruction: long-term experience with an algorithm for laser endopyelotomy and laparoscopic retroperitoneal pyeloplasty. J Urol 2007; 177: 1000-5.

16. Acher PL, Nair R, Abburaju JS, et al. Ureteroscopic holmium laser endopyelotomy for ureteropelvic junction stenosis after pyeloplasty. J Endourol 2009; 23: 899-902.

17. Di Grazia E, Nicolosi D. Ureteroscopic laser endopyelotomy in secondary UPJ obstruction after pyeloplasty failure. Urol Int 2005; 75: 333-6.

18. Hibi H, Ohori T, Taki T, et al. Long-term results of endoureterotomy using a holmium laser. Int J Urol 2007; 14: 872-4.
19. Doo CK, Hong B, Park T, Park HK. Long-term outcome of endopyelotomy for the treatment of ureteropelvic junction obstruction: how long should patients be followed up? J Endourol 2007; 21: 158-61.

20. Davis DM. Intubated ureterotomy: a new operation for ureteral and ureteropelvic strictures. Surg Gynecol Obstet 1943; 76: 513. 